\title{
Detection efficiency of the Solar Neutron Telescopes located at high altitudes
}

\author{
L.X. González ${ }^{a}$, J.F. Valdés-Galicia ${ }^{b}$, Y. Murakic ${ }^{c 1}$, K. Watababe ${ }^{d}$, T. Sako ${ }^{c}$, K. Koga ${ }^{d}$, \\ Y. Matsubarac ${ }^{\text {, K. Kamiya }}{ }^{\mathrm{d}}$, S. Shibata ${ }^{\mathrm{e}}$, T. Sakaif. \\ ${ }^{a}$ LANCE-SCiESMEX, Instituto de Geofísica-UM. Universidad Nacional Autónoma de México, Morelia. \\ 58190, Michoacán, México.E-mail:xavier@geofisica.unam.mx \\ ${ }^{b}$ Instituto de Geofísica, UNAM. Av. Universidad 3000, CU. 04510, Coyoacán, Ciudad de México, \\ México. \\ ${ }^{c}$ Institute for Space-Earth Environmental research, Nagoya University. Nagoya 464-8601, Japan. \\ ${ }^{d}$ ISAS, JAXA, Sagamihara 229-8510, japan. \\ ${ }^{e}$ Engineering Science laboratory, College of Engineering, Chubu University, Kasugai, Japan. \\ ${ }^{f}$ College of Industrial Technologies, Nihon University, Narashino 275-0005, Japan.
}

\begin{abstract}
Four solar neutron telescopes (SNTs) are installed at high altitude in China (Tibet, $4300 \mathrm{~m}$ a.s.1.), (Japan, Mt Norikura, $2770 \mathrm{~m}$ a.s.1), Mexico (Mt. Sierra Negra, $4850 \mathrm{~m}$ a.s.l) and Bolivia (Mt. Chacaltaya, $5250 \mathrm{~m}$ a.s.l). The SNT are composed by plastic scintillators (PS), photomultipliers tubes (PMT) are set on top of the PS. Proportional counters (PRC) surround the detector to discriminate between charged and neutral particles by anti-coincidence signal. Underneath the PS of the SNT an array of PRC is set to determine the arrival direction. The energy discriminator thresholds of the PMT are set at different energy levels in function of the PS thickness. The thickness of the PS is $40 \mathrm{~cm}$ (Tibet), $20 \mathrm{~cm}$ (Norikura), $30 \mathrm{~cm}$ (Sierra Negra) and $40 \mathrm{~cm}$ (Chacaltaya). Incoming particles produce recoil protons by nuclear interactions with the PS. The PMT measure the ionization energy loss of the protons. In this way, the SNT can discriminate between incident charged and neutral particles, measure the energy, and determine the direction of the incoming particles. In this work, we present the experimental, GEANT3 and GEANT4 simulation results of the detection efficiency of the SNT to solar neutrons impinging the top of the detectors.
\end{abstract}

35th International Cosmic Ray Conference - ICRC2017

10-20 July, 2017

Bexco, Busan, Korea

${ }^{1}$ Speaker

(C) Copyright owned by the author(s) under the terms of the Creative Commons Attribution-NonCommercial-NoDerivatives 4.0 International License (CC BY-NC-ND 4.0). 


\section{Introduction}

Solar neutrons are released by nuclear reaction between accelerated ions in the solar atmosphere during the X-class flare [1]. Since neutrons are not affected by interplanetary magnetic fields, it is important to be able to detect them at ground level, because they preserve key information about acceleration mechanism in the solar flare. To measure solar neutrons with ground detectors, the neutrons need to be very energetic, because the lifetime of free solar neutrons is $885 \mathrm{~s}$; the detectors must be located at high altitudes and near the equator, because the neutrons are strongly attenuated in the Earth's atmosphere [2], and to have a high rigidity cutoff to exclude the low energy solar ions produced in the same flare as neutrons. Furthermore, there is an asymmetry in the distribution of neutrons caused by the refraction effect in the atmosphere [3].

The neutron monitors (NMs) were designed to detect the background of galactic cosmic rays, where the time and energy resolution are not critical. The NMs have high sensitivity and they cannot recognize the arrival direction of the impinging particles; therefore, the NMs are not the best detectors to measure solar neutrons.

The solar neutron telescopes (SNTs) have the capability to measure the arrival direction and the deposited energy of particles, as well as to discriminate between charged and neutral particles. The solar neutron telescope worldwide network is composed by seven telescopes (Table 1), but for this paper, we will take into account four SNTs installed in Bolivia, China, Japan and Mexico.

\section{Solar Neutron Telescopes (SNTs)}

On the basis that the SNT would be the ideal instrument to detect neutrons emitted in the flares, in 1991 a $1 \mathrm{~m}^{2}$ prototype was installed at Norikura, Japan. The technique developed has been improved as new, more sophisticated versions were produced in later years. The latest SNT version was installed in Sierra Negra, Mexico (see Table 1).

The main idea is to use plastic scintillators (PS) to estimate the energy of the incoming particles by measurements of the photon emitted by recoil protons produced by the interaction with $\mathrm{H}$ and $\mathrm{C}$ nuclei in the PS. On top of the PS there are photo-multipliers tubes (PMTs). As anti-signal or veto-counters the PS are surrounded by proportional counters (PRC) or scintillator detectors, in this way, a charged particle releases two signals (called: without_anti) and the neutral particles release one signal (called: with_anti) in the anti-coincidence circuits. 
To determine the arrival direction of the incoming particles, underneath of the some SNTs is located an array of PRC that can detect the recoil protons produced in the PS, that are scattered in the direction of the incident particles.

\begin{tabular}{|ccccccccc|}
\hline \multicolumn{7}{c}{ Table 1. Solar Neutron Telescope Worldwide Network } \\
\hline Location & Long & Lat & $\begin{array}{c}\text { Height } \\
{[\mathrm{m} \text { a.s.l. }]}\end{array}$ & $\begin{array}{c}\text { Air mass } \\
{\left[\mathrm{g} / \mathrm{cm}^{2}\right]}\end{array}$ & $\begin{array}{c}\text { Area } \\
{\left[\mathrm{m}^{2}\right]}\end{array}$ & $\begin{array}{c}\text { PS thickness } \\
{[\mathrm{cm}]}\end{array}$ & $\begin{array}{c}\text { Operation } \\
{[\text { Since }]}\end{array}$ \\
\hline Chacaltaya, Bolivia & $68^{\circ} \mathrm{W}$ & $16.2^{\circ} \mathrm{S}$ & 5250 & 540 & 4 & 40 & Sep, 1992 \\
Norikura, Japan & $137.5^{\circ} \mathrm{E}$ & $36.1^{\circ} \mathrm{N}$ & 2770 & 730 & 64 & 20 & Oct, 1996 \\
Mauna Kea, USA & $156.3^{\circ} \mathrm{W}$ & $19.8^{\circ} \mathrm{N}$ & 4200 & 610 & 8 & 20 & Apr, 1997 \\
Gornergrat, Switzerland & $7.8^{\circ} \mathrm{E}$ & $46.0^{\circ} \mathrm{N}$ & 3135 & 690 & 4 & 40 & Jan, 1998 \\
Yangbajing, China & $90.5^{\circ} \mathrm{E}$ & $30.0^{\circ} \mathrm{N}$ & 4300 & 600 & 9 & 40 & Sept, 1999 \\
Aragats, Armenia & $40.5^{\circ} \mathrm{E}$ & $44.2^{\circ} \mathrm{N}$ & 3200 & 670 & 4 & 60 & Feb, 2003 \\
Sierra Negra, Mexico & $97.3^{\circ} \mathrm{W}$ & $19.0^{\circ} \mathrm{N}$ & 5580 & 575 & 4 & 30 & Nov, 2003 \\
\hline
\end{tabular}

The energy deposited $\left(\mathrm{E}_{\mathrm{dep}}\right)$ by particles in the PS is measured by PMTs and it is discriminated in energy thresholds in function of the PS thickness. $\mathrm{E}_{\mathrm{dep}}>40 \mathrm{MeV},>80 \mathrm{MeV}$, $>120 \mathrm{MeV}$ and $>160 \mathrm{MeV}$ for the SNTs in Bolivia and China. $\mathrm{E}_{\text {dep }}>20 \mathrm{MeV},>40 \mathrm{MeV},>80$ $\mathrm{MeV},>120 \mathrm{MeV}$ and $>160 \mathrm{MeV}$ for the SNT in Japan. $\mathrm{E}_{\mathrm{dep}}>30 \mathrm{MeV},>60 \mathrm{MeV},>90 \mathrm{MeV}$ and $120 \mathrm{MeV}$ for the SNT in Mexico.

\section{Efficiency of the Solar Neutrons Telescopes (SNTs)}

In order to know the detection capability of the SNTs for different particle species, in particular solar neutrons, the efficiency of the SNTs has been calculated by Geant3 (China, Bolivia and Japan), with an accelerator experiment (Bolivia and Japan) and GEANT4 Monte Carlo simulations [4] (Bolivia and Mexico). For the four SNTs analyzed in this work, we have simulated all the detector active components and take into account all the physical processes relevant to particles passing through matter. Default and different cross-section sets are provided automatically for each type of hadronic process, particles and materials.

For the SNT installed at Yangbajing, China, the efficiency is shown in figure 1; the total area is $9 \mathrm{~m}^{2}$ and the neutrons were injected with a zenith angle of $0^{\circ}$. Figure 2 shows the results of the efficiency for the $64 \mathrm{~m}^{2}$ of PS SNT installed at Mt. Norikura, Japan. The left plot shows the experimental results (black points) obtained by accelerator and the GEANT3 simulation results (white points) for four energy threshold channels. The right plot represents the GEANT3 
simulation results for the five energy channels. The solar neutrons were injected with $12^{\circ}$ of zenith angle. In figure 3, we show the solar neutron detection efficiency for the SNT installed at Chacaltaya, Bolivia. The left plot shows the combined results of experimental data by accelerator (black points) and GEANT3 simulations (white points), and the right plot shows the relationship between the incident energy of solar neutrons at the top of the detector and the energy deposited in the PS for $0^{\circ}, 30^{\circ}$ and $60^{\circ}$ zenith angles, estimated by GEANT4 simulations. In the left panel of figure 4, we show the probability of detection of the four channels of energy deposition of the SNT at Sierra Negra, Mexico, in function of the incident energy of the solar neutrons at the top of the detector, using GEANT4 simulations. The right panel of figure 4 shows simulation results of the energy deposited by the impinging solar neutrons in the PS, in function of the incident energy of neutrons at the top of the SNT.

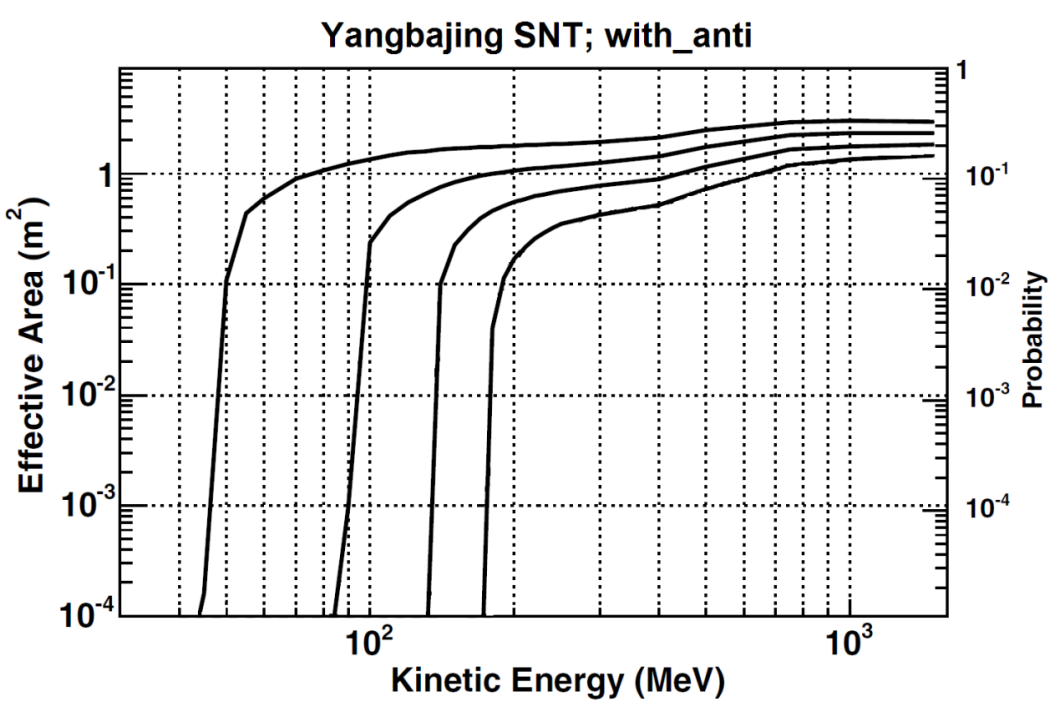

Figure 1. Detection efficiency of the Yangbajing SNT for solar neutrons. The lines indicate the deposition energy $\left(E_{\text {dep }}\right)$ of the four energy thresholds channels, $E_{\text {dep }}>40 \mathrm{MeV},>80 \mathrm{MeV},>120 \mathrm{MeV}$ and $>160 \mathrm{MeV}$, [5]. The left axis shows the effective area of the detector. 

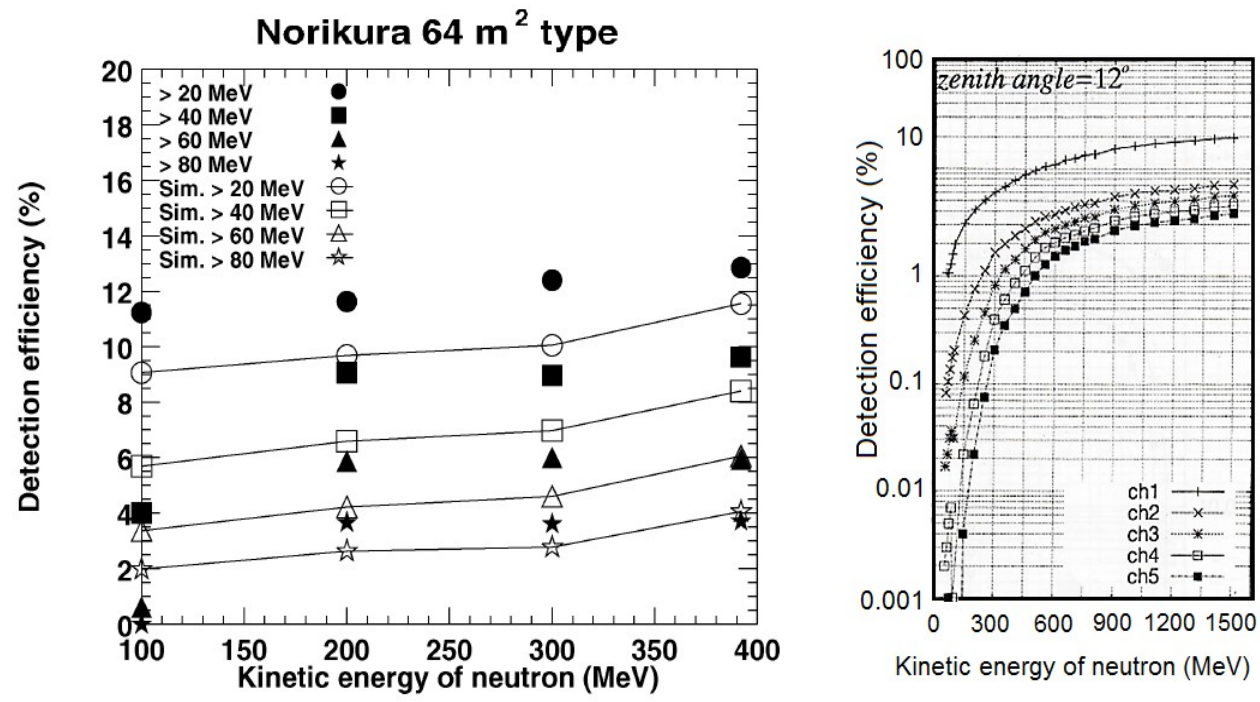

Figure 2. Solar neutron detection efficiency of the Norikura, Japan SNT. The left plot shows the experimental results of the $64 \mathrm{~m}^{2}$ of PS using an accelerator, [6]. The right plot shows the GEANT3 simulation results for the five energy threshold channels with injection zenith angle of $12^{\circ}$.
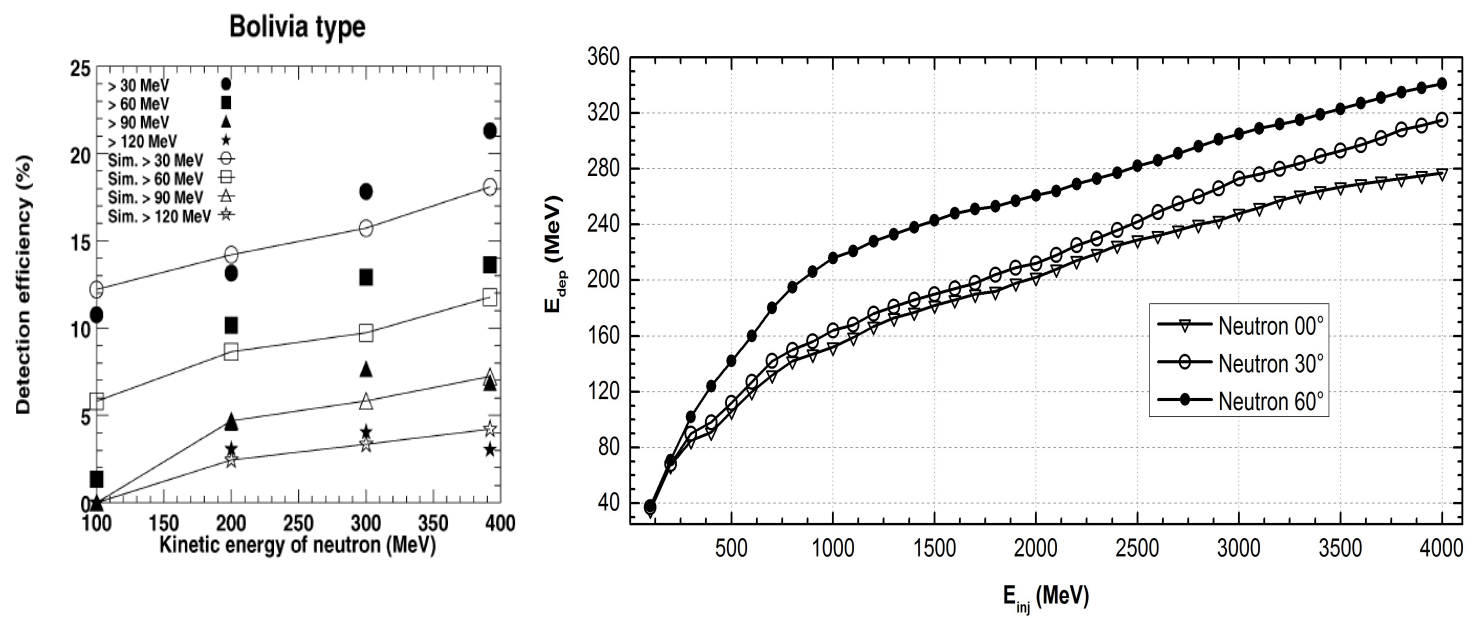

Figure 3. Detection efficiency of the Chacaltaya, Bolivia SNT for solar neutrons. In the left plot, the black points show the experimental results for four energy thresholds channels, $E_{\text {dep }}>30 \mathrm{MeV},>60 \mathrm{MeV}$, $>90 \mathrm{MeV}$ and $>120 \mathrm{MeV}$, using an accelerator, and the results of GEANT3 simulation are given by white points [6]. The right panel shows the energy deposited by the impinging solar neutrons in the scintillator as a function of their incident energies at the top of the detector for three zenith angles, using GEANT4, [7]. 

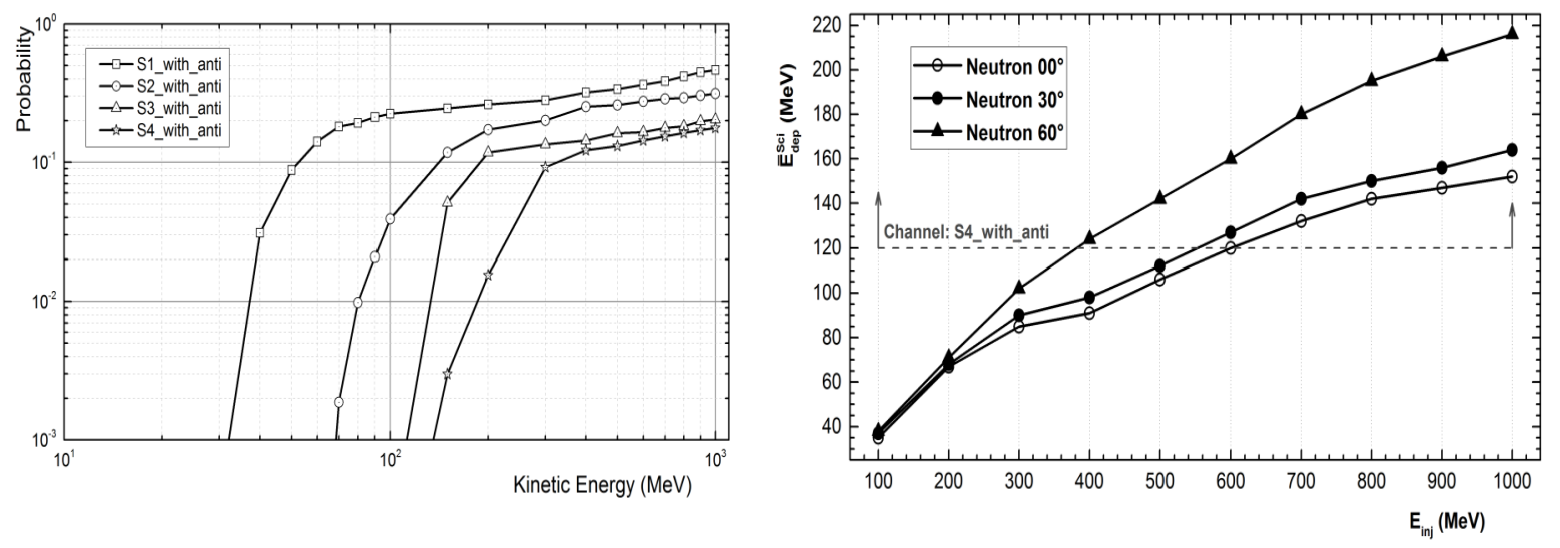

Figure 4. Detection efficiency of the Sierra Negra, Mexico SNT for solar neutrons. The left panel shows the response of the four energy threshold channels (S1_with_anti: $>30 \mathrm{MeV}$, S2_with_anti: $>60 \mathrm{MeV}$, S3_with_anti: $>90 \mathrm{MeV}$ and S4_with_anti: $>120 \mathrm{MeV}$ ) of the detector according to the GEANT4 simulations. The probabilities of detection for each channel are calculated as a function of the energy of the neutrons at the top of the SNT. The neutrons were distributed evenly over the detector with a vertical injection angle ( $0^{\circ}$ zenith). The right panel shows the relationship between the primary energy of neutrons and the energy deposited by these neutrons in the PS for three different zenith angles, [7].

\section{Conclusions}

We know the solar neutron detection efficiency of the Solar Neutrons Telescopes. The results of the simulation and experimental data are in good agreement. On the basis that the SNT is the best detector to measure solar neutrons, we developed GEANT4 Monte Carlo simulation codes to know the response of the SNTs and their energy threshold channels. The SNTs are ready to detect a solar neutrons event.

\section{References}

[1] R.E. Lingenfelter, et al., 1965. J Geophys Res 70:4087-4095

[2] S. Shibata, et al., 1994. J. Geophys. Res. 99, 6651-6665.

[3] L.I. Dorman, et al., J. Geophys. Res. 495, (1999) 1634.

[4] S.Agostinelli, et al., Nucl. Instr. and Meth. A 506, (2003).

[5] Y. Muraki, et al., Astro. Phys. 28 (2007), 119-131.

[6] H. Tsuchiya, et al., Nucl. Instr. and Meth. A 463, (2001).

[7] L.X. González, et al., ApJ. 814:136, 2015. 